\title{
Diesel Cooling System Modeling for Electrification Potential
}

\author{
John Batteh ${ }^{1}$ Ashok Kumar Ravi ${ }^{2}$ Dale Pickelman ${ }^{3}$ \\ ${ }^{1}$ Modelon Inc., USA, john. batteh@modelon.com \\ ${ }^{2}$ Modelon Engineering Private Limited, India, ashokkumar. ravi@modelon. com \\ ${ }^{3}$ Hanon Systems, USA, dpickelmehanonsystems . com
}

\begin{abstract}
Electrification of automotive systems presents significant opportunities for improvements in cooling system efficiency and performance. This paper describes an effort to develop an analytic platform for Hanon Systems to evaluate the electrification potential for powertrain cooling systems. The paper describes the development of a baseline diesel cooling system model based on the Ford 6.7L Power Stroke diesel. A variant of the system with electric pumps is also modeled. Performance of the baseline conventional and electric pump system are compared on a typical automotive drive cycle to quantify potential benefits of the electric pump system and advanced controls.
\end{abstract}

Keywords: cooling systems, diesel, electrification

\section{Introduction}

Electrification is a pervasive trend in the auto industry, from fully electric vehicles to hybrids to electrification of individual subsystems and components. For all powertrain systems, thermal management of the components is a critical requirement for the safe and efficient operation of the system. Furthermore, thermal constraints for electric powertrains can limit performance (Stellato, 2017). Significant energy is required to pump cooling fluid for thermal management. Though varying with engine, cycle/operating conditions, fuel type, system design, etc., 1-3\% of fuel energy can be consumed by pumps for cooling and lubrication systems (Thiruvengadam, 2014).

In conventional cooling systems, mechanical pumps are driven by the engine. Connected through a fixed drive ratio to the engine, mechanical pumps operate based on engine speed. Since the flowrate is linked to the engine speed, sizing of the pumps for mechanical systems for maximum cooling load can be problematic. In many vehicles, maximum cooling load results from operating conditions with high engine load and potentially low engine speed and vehicle speed/external air flow. To meet this maximum demand, the requirement drives a large pump size. With the linking of pump speed to engine speed, significant inefficiencies can result from mismatch in pump efficiency to typical operating conditions, overflow in the system, and potentially even over cooling under some conditions.

Replacing a mechanical pump with an electric pump can yield significant benefits. With the pump speed decoupled from the engine speed, the electric pump can be sized more appropriately to meet cooling system demand. Decoupling from the engine operation also means that the electric pump speed can be controlled to provide flowrates on demand to better match the cooling load demand. Advanced control strategies can also lead to additional benefits by optimizing warm up for lubricating fluids like engine oil and transmission oil. Previous analytic studies have demonstrated potential benefits of $1.2 \%$ for electric pump systems with advanced controls on a vehicle driven by a $1 \mathrm{~L}$ turbo gas direct injection engine with additional benefits due to optimized transmission thermal conditions (Zheng, 2018). While careful design of the system is required to fully realize these efficiency improvements, there is clearly motivation to pursue given the potential impact on fuel economy or electric range.

System modeling with Modelica has been widely used for vehicle thermal management simulations (Bouvy, 2012; Krüger, 2012; Batteh, 2014; Stellato, 2017). With a powerful and flexible modeling framework and proven commercial libraries, Modelica provides an ideal platform for architectural studies and controls prototyping for advanced vehicle thermal management. This paper describes an effort to develop an analytic platform for Hanon Systems to evaluate the electrification potential for powertrain cooling systems. The goal of this analytic platform is to allow rapid virtual prototyping of different cooling systems for evaluation of the potential of Hanon hardware and controls solutions for system optimization. To demonstrate this platform, a model of a diesel cooling system based on a Ford Power Stroke diesel 6.7L V8 was developed. A baseline model of the system is developed with Liquid Cooling Library (Modelon AB, 2018) and then modified to include electric pumps from Hanon. The results from the simulations are compared on a typical automotive drive cycle to quantify potential benefits of the electric pump system. 


\section{Diesel Cooling System Model}

This section provides an overview of the diesel cooling system model. The model is based on the Ford Power Stroke (code name "Scorpion") 6.7L V8 diesel designed for the North American light commercial truck market (Deraad, 2010). The Power Stroke diesel is used in Ford F series and Super Duty pickup trucks. The Ford Scorpion diesel was designed with an innovative dual loop cooling system. The following sections provide an overview of the full system model and relevant component modeling details.

\subsection{System and Model Overview}

The Scorpion diesel system has two cooling loops: a high temperature loop and a low temperature loop. The two circuits are completely unmixed and interact with each other via the two stage EGR cooler and via the radiator air flow with the low temperature radiator in front of the high temperature radiator. The high temperature loop provides cooling and coolant flow for the following components:

- Engine block and head

- Turbocharger

- EGR cooler $\left(1^{\text {st }}\right.$ stage $)$

- Heater core

- Engine oil cooler

The low temperature loop provides cooling and coolant flow for the following components:

- EGR cooler ( $2^{\text {nd }}$ stage $)$

- Transmission oil cooler

- Charge air cooler

- Fuel cooler

Figure 1 shows the entire diesel cooling system model. The model was built without substantial system hierarchy per request when getting started with Modelica-based modeling. The model was built based on publicly available information on the system, including the service manual for the engine and cooling system. Characterization of the model is discussed in Section 2.2. To help understand the coolant flow and system operation, a discussion of relevant sections of the model follows.

The high temperature loop operates at typical coolant operating temperatures around $100^{\circ} \mathrm{C}$. Starting from the high temperature pump, the coolant flow splits between the EGR cooler and the engine. The flow through the engine goes to the left and right block and head. Some flow from the left side is sent to the turbocharger. Some flow from the right side of the engine is sent to the engine oil cooler. The flow from the EGR cooler, turbocharger, and resulting flow through the engine join downstream before the thermostat. Some flow goes to the heater core while the remaining flow goes through the thermostat. The thermostat controls the balance of flow between the radiator and the bypass. The heater core flow merges downstream of the radiator and then flows to the degas bottle. The resulting flow is mixed with the bypass and flow from the engine oil cooler and then flows to the pump.

The low temperature loop is a fairly complex hydraulic circuit with multiple flow branches and operating modes based on coolant temperature. The low temperature loop also has operation at higher temperatures and at lower temperatures. The higher temperature portion of the system operates at coolant temperatures greater than $45^{\circ} \mathrm{C}$ and maintained approximately at $60^{\circ} \mathrm{C}$. The lower temperature part of the circuit operates at coolant temperatures greater than $20^{\circ} \mathrm{C}$ and maintained approximately at $45^{\circ} \mathrm{C}$. Starting from the pump, some of the flow splits to the upper section of the radiator and the other part of the flow goes to a high temp thermostat that can direct flow to the EGR cooler second stage and the transmission oil cooler. When the coolant temperature is below $45^{\circ} \mathrm{C}$, the high temp thermostat is closed, and some of the flow is sent directly to the transmission oil cooler and EGR cooler. When the coolant temperature is above $45^{\circ} \mathrm{C}$, the high temp thermostat starts to open and flow to the EGR cooler and transmission oil cooler is extracted after passing through the radiator upper section. The low temperature thermostat is located in the radiator tank. This thermostat controls the flow between the upper and lower sections of the radiator. When the coolant entering the radiator is less than $20^{\circ} \mathrm{C}$, the radiator is bypassed altogether, and the flow is directed to the fuel cooler and charge air cooler. When the coolant reaches $20^{\circ} \mathrm{C}$, the low temperature thermostat starts to open and allows coolant flow through the upper and low sections of the radiator before flowing to the charge air cooler and fuel cooler. Recall that the flow to the EGR cooler and transmission oil cooler can be extracted after the radiator upper section. Under different operating conditions, it is possible to bypass the radiator altogether, use only the upper section of the radiator, or use both the upper and lower sections of the radiator. To allow this capability, the two sections of the radiator are modeled as separate heat exchangers. The flow from all coolers joins at the degas bottle upstream of the pump.

The Scorpion diesel system provides a nice benchmark for an analytic platform for virtual prototyping of hardware and control strategies given its overall complexity with multiple loops, multiple coolers, and multiple different temperature levels. 


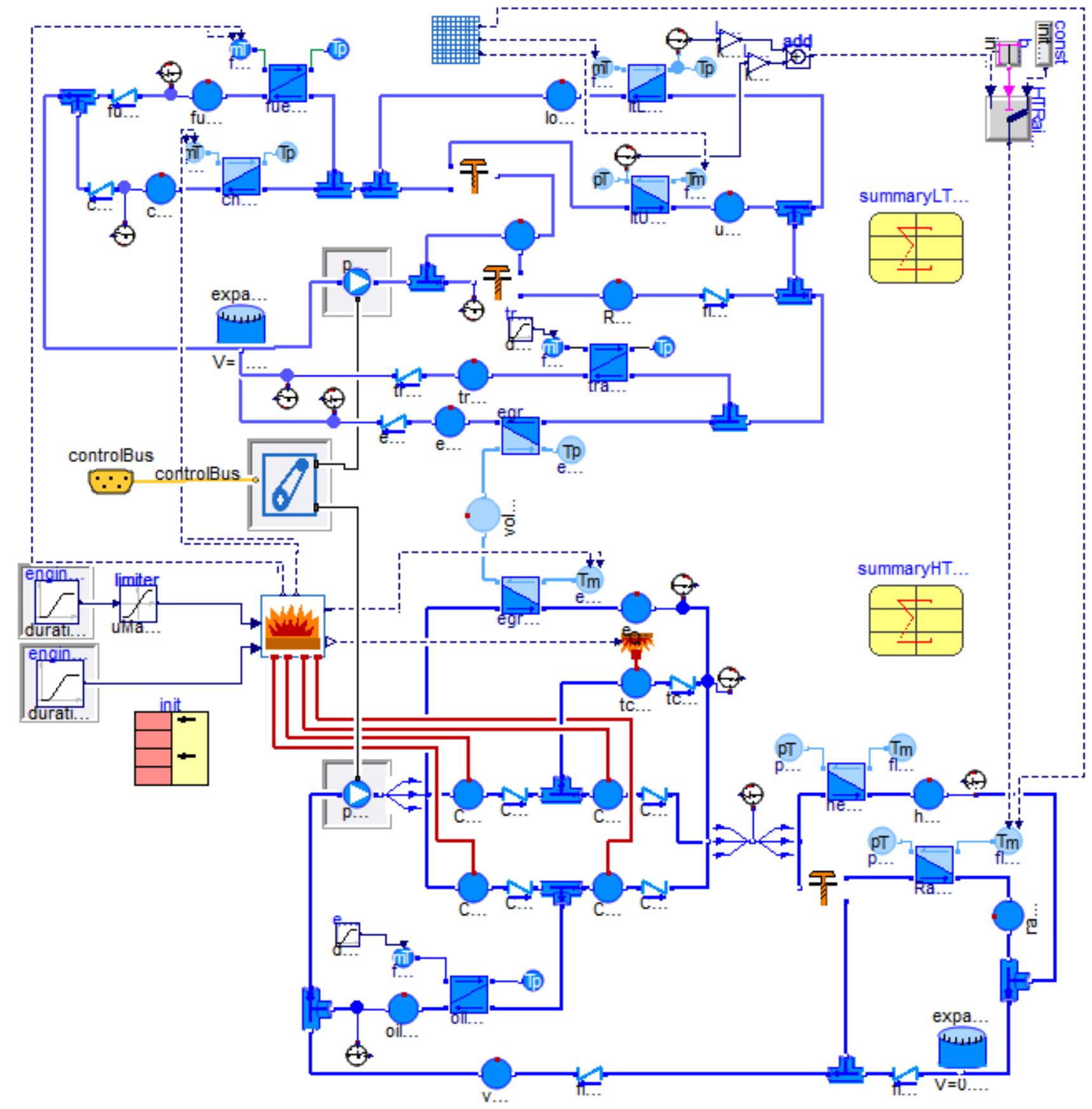

Figure 1. Diesel cooling system model based on the Ford Power Stroke diesel 6.7L V8 with high temperature and low temperature circuits 
In addition to the coolant hydraulic circuits, simple hydraulic circuits are modeled for the transmission oil and engine oil. Figure 2 shows this simple circuit for the engine oil cooler and also the implementation of the simple circuit. The inputs to the circuit are the oil flowrate through the cooler and the heat input to the oil and are considered boundary conditions. With this simple circuit, it is possible to simulate dynamic oil temperatures including warmup. While more detailed models can be built if information is available to do so, it is important to have dynamic estimates of oil temperature to ensure that oil temperature limits are respected when considering system variants and also to estimate potential benefits of faster oil temperature warmup for friction reduction.

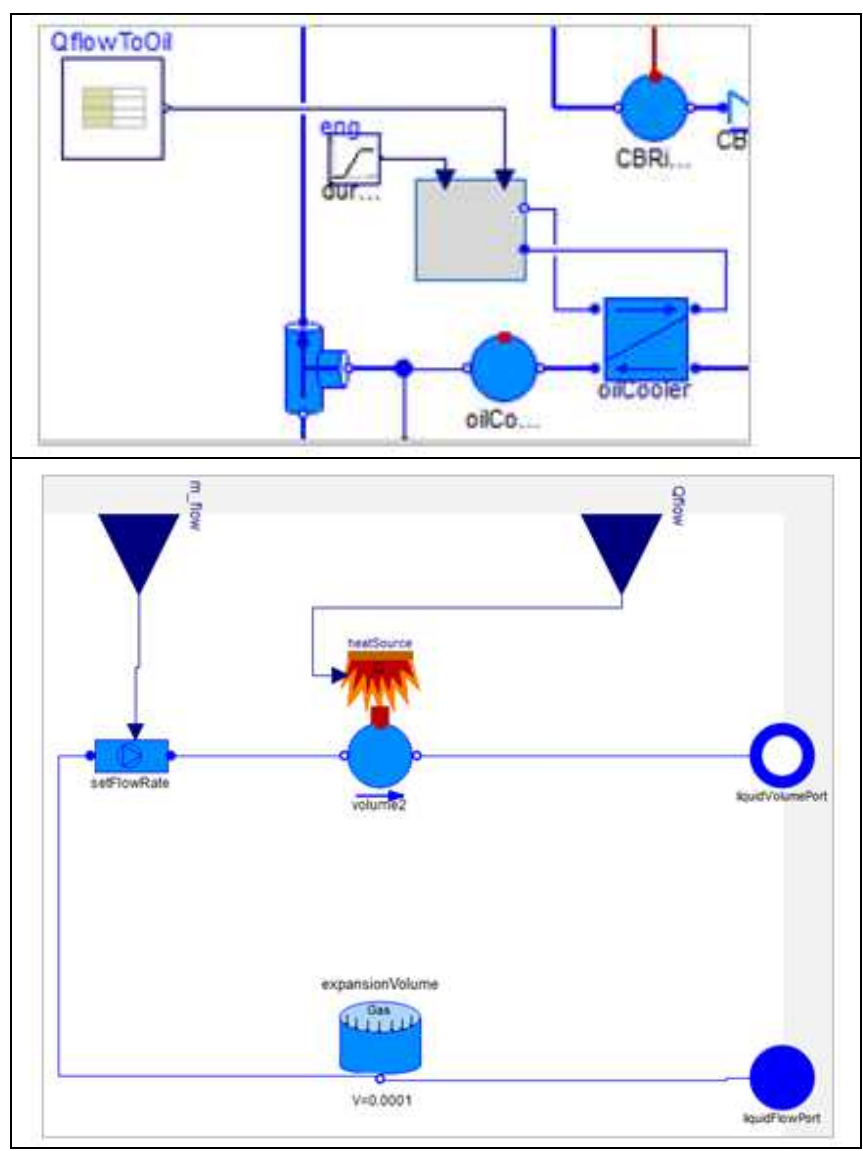

Figure 2. Simple engine oil circuit

Several configurable elements are included in the model to provide the flexibility to switch between the mechanical pump system and the electric pump system. The pump models in the high temperature and low temperature circuits are replaceable models and can be changed individually. A control bus structure is also established. Sensor signals from relevant components are placed onto the control bus. A controller component is then connected to the coolant pumps to specify the pump speed. For the mechanical pump system, the engine speed is passed through to the mechanical pumps offset by a fixed ratio. For the electric pump system, controllers are implemented as discussed in subsequent sections.

The focus of the system model is on the cooling system, but obviously simulation of the cooling system is not possible without the relevant heat inputs and boundary conditions. While a full vehicle simulation can provide some of these inputs, that scope was outside of the focus of the current effort. To support the cooling system modeling effort, a simple mapbased engine shown in Figure 3 was developed to estimate heat input from the engine and turbocharger along with operation conditions (flow and temperatures) for the EGR cooler, fuel cooler, and charge air cooler as a function of engine operating conditions. The inputs to the model are the engine brake power and engine speed. The brake specific fuel consumption (BSFC) map was used to calculate fuel flow based on published data (Deraad, 2010). Figure 4 shows the BSFC map as extracted. While highly simplified, this engine model allows simulation of the key inputs from the engine without requiring highly detailed information on the engine and engine operating conditions. Data for this model was input based on some published operating conditions (Deraad, 2010) and then supplemented with nominal information for diesel engines. Predictive capability of this model would obviously be improved with actual engine characterization data, but the basic model does provide a practical computational approach for the engine in lieu of detailed engine mapping data.

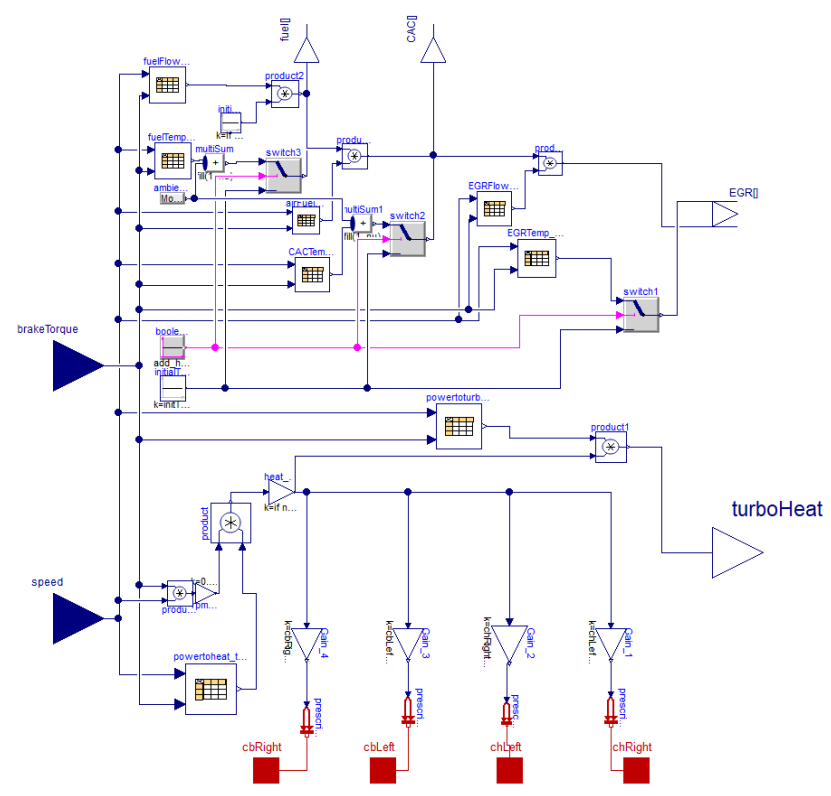

Figure 3. Map based engine model 


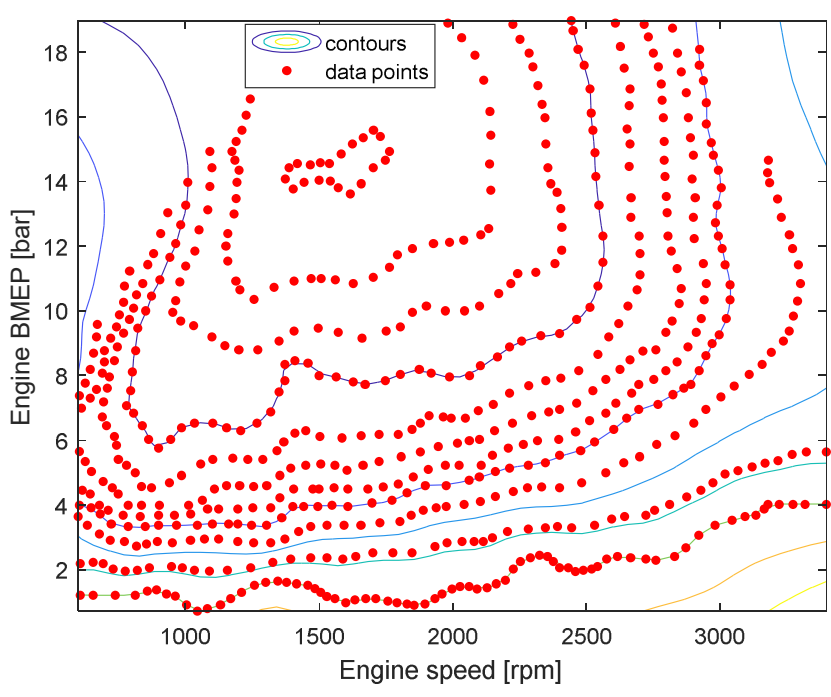

Figure 4. Engine BSFC map as extracted from reference (Deraad, 2010)

In conjunction with the engine model, the boundary conditions for the system are as follows:

- Engine brake power

- Engine speed

- Vehicle speed

- Heat input and flow for engine oil

- Heat input and flow for transmission oil

- Heater core air temperature and flowrate

- Inlet air temperature of low temperature radiator

An initialization component is included with the system model to allow convenient, consistent initialization of the system and specification of the boundary conditions. The initialization component also includes the ability to override the engine calculations to allow isothermal simulations and simulation of the system at specified steady state boundary conditions.

Summary records are included for both the high temperature and low temperature circuits to allow easy access to relevant outputs, including flowrates, temperatures, heat rejection in the coolers, etc.

\subsection{System Characterization}

One of the main challenges in building the model of the Scorpion diesel cooling system is the lack of data to parameterize the components and characterize the system outside of publicly-available data in literature. This data along with knowledge of similar systems was used to get a reasonable, first cut system model though is admittedly imperfect and not desirable for model accuracy.

One key piece of characterization data is the flow and efficiency data for the mechanical pump. This data was not readily obtained from published literature. Hanon provided estimates of the flow and system head requirements for the circuits. This data was then used to characterize the mechanical pump model at different operating speeds. No mechanical efficiency data was provided so the pump was assumed to operate at a constant $55 \%$ efficiency. This value could be considered on the high side, but a conservative value was chosen so as to not bias results towards the electric pumps. For the calculation of energy to drive the pump, the fuel power required to drive the pump was calculated using the engine BSFC.

For the electric pump system variant, Hanon provided estimates of the pump efficiency and flowrates vs. head characteristics at various speeds based on development and actual hardware. The flow characteristics for the $\mathrm{HCP}-1 \mathrm{KW}$ pump used in the high temperature circuit are shown in Figure 5. The efficiency data provided mapped from hydraulic power to electric power. Since the electric pump system is included in a conventional system and to provide consistent comparisons of energy to drive the pump, the fuel power required to drive the electric pumps was calculated based on the efficiency data provided and an estimate of the alternator efficiency along with the engine BSFC. The alternator efficiency was assumed to be $55 \%$ at all operating conditions. With this approach, fuel energy comparisons can be made between the mechanical and electric pump systems.

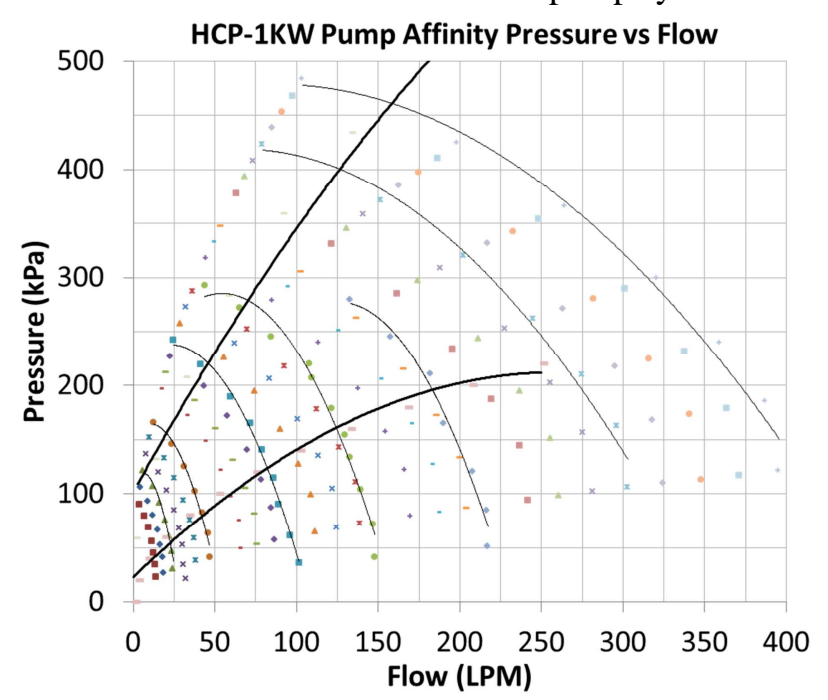

Figure 5. Flow characteristics for Hanon HCP-1KW pump used in high temperature cooling loop

Since no detailed flow information was available for the individual flow branches at different conditions, assumptions were made based on information for a few operating points to establish a flow distribution. The cooling system service manual provided information on the thermostat opening conditions for both the high temperature and low temperature circuits. As can be seen in Figure 1, lumped flow resistances were included in the various branches to allow calibration of the flow distribution. The flow resistances are parameterized with an operating point friction model that takes nominal flow and pressure drop data for a given operating point. Using information for the 
thermostats, isothermal flow tests were conducted at various operating conditions to calibrate the flow resistances in the model to provide the desired flow distribution. The flow characterization of the system remains fixed and only the pumps are switched to go from the mechanical to electric pump system.

The system volume for the high temperature and low temperature circuits were provided in the cooling system manual. The distribution of the volume within the individual circuit was provided based on rough judgement of the sizes of the various components. The volume of the oil circuits was also obtained based on available data and entered into the simplified circuits.

Following the flow characterization of the system, thermal characterization of the system is required to run reasonable thermal simulations. Typically this sort of characterization is easily provided based on heat exchanger performance characteristics that can be readily entered into the model. The heat exchangers in the system shown in Figure 1 include the high temperature radiator, heater core, engine oil cooler, low temperature radiator broken into upper and lower sections, EGR cooler broken into high temperature and low temperature sections, transmission oil cooler, fuel cooler, and charge air cooler. These heat exchangers are all modeled using the StaticEffectivenessTable model in Liquid Cooling Library. This model specifies the heat exchanger performance as a $2 \mathrm{D}$ effectiveness table based on the mass flowrates of the individual fluid streams. However, this sort of data was not readily available in public literature. To provide characterization data for the heat exchangers, Hanon provided data for similar types of heat exchangers based on simulated and actual hardware. This data was then scaled as needed to provide the effectiveness maps used in the model. The heater core was not characterized but was set to an inactive state in the model.

To characterize the radiator airflow, the flow areas to the high temperature radiator and the two sections of the low temperature radiator were estimated. A table for the grill factor as a function of vehicle speed was estimated. Using this table, the external air velocity was calculated. This velocity was then converted to a mass flow using the flow area parameters. Since the low temperature radiator is in front of the high temperature radiator, the external air outlet temperature from the low temperature radiator is used as the inlet temperature for the high temperature radiator. While manually considered in this model, detailed heat exchanger models using Heat Exchanger Library (Modelon AB, 2018) handles this stacking effect in a natural, distributed way based on component geometry and stack layout. These models could be integrated into the cooling system circuit but were beyond the scope of this effort given that they require designoriented geometric data not readily available.

\subsection{Electric Pump Control}

The conventional mechanical system operates without active control for the coolant flow as the pump speeds are determined by the engine, and thermostats are passive flow control devices based on operational setpoints. For the system retrofit with electric pumps, a controller is implemented to control the pump speed. Figure 6 shows the electric pump controller. The controller operates based on target coolant temperatures for the various coolers. The commanded pump speed increases as coolant temperatures exceed the target temperatures. A minimum pump speed is specified to ensure that there is sufficient flowrate to avoid hotspots in the system. In addition, the pump hardware is designed to only operate in a particular speed range. A maximum pump flowrate is also specified to ensure that the pump stays within operational limits.

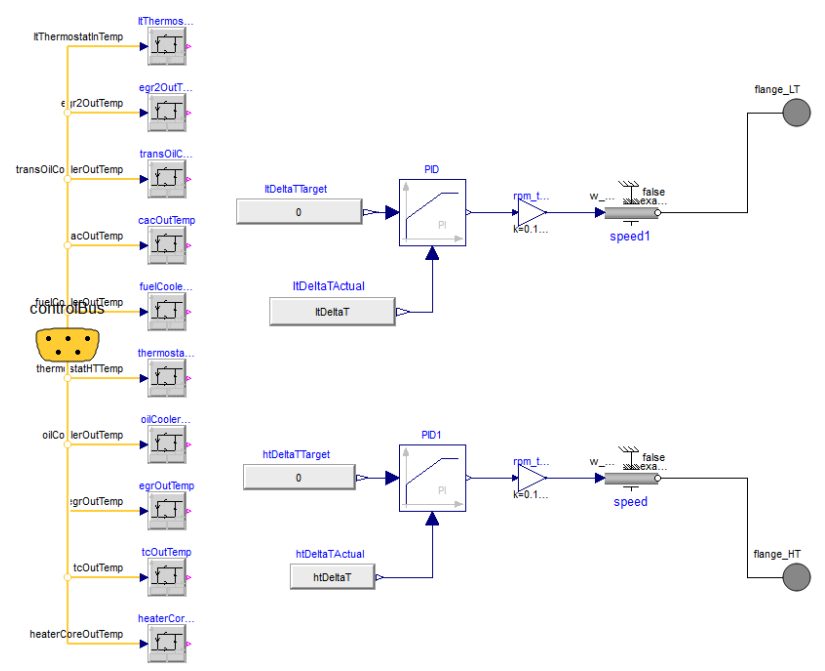

Figure 6. Electric pump controller

\section{Simulation Results}

Following the characterization of the system, a series of simulations were run to evaluate the potential of the electric pump system as compared to the baseline mechanical pump system. The simulations were conducted on the FTP cycle. Figure 7 shows operating conditions for the FTP cycle used for the simulations including the following plots from top to bottom:

- Vehicle speed [kph]

- Engine speed [rpm]

- Engine power [W]

- Fuel flow rate $[\mathrm{g} / \mathrm{s}]$

- High temperature radiator flow rate $[\mathrm{kg} / \mathrm{s}]$

- Low temperature radiator flow rate $[\mathrm{kg} / \mathrm{s}]$ 


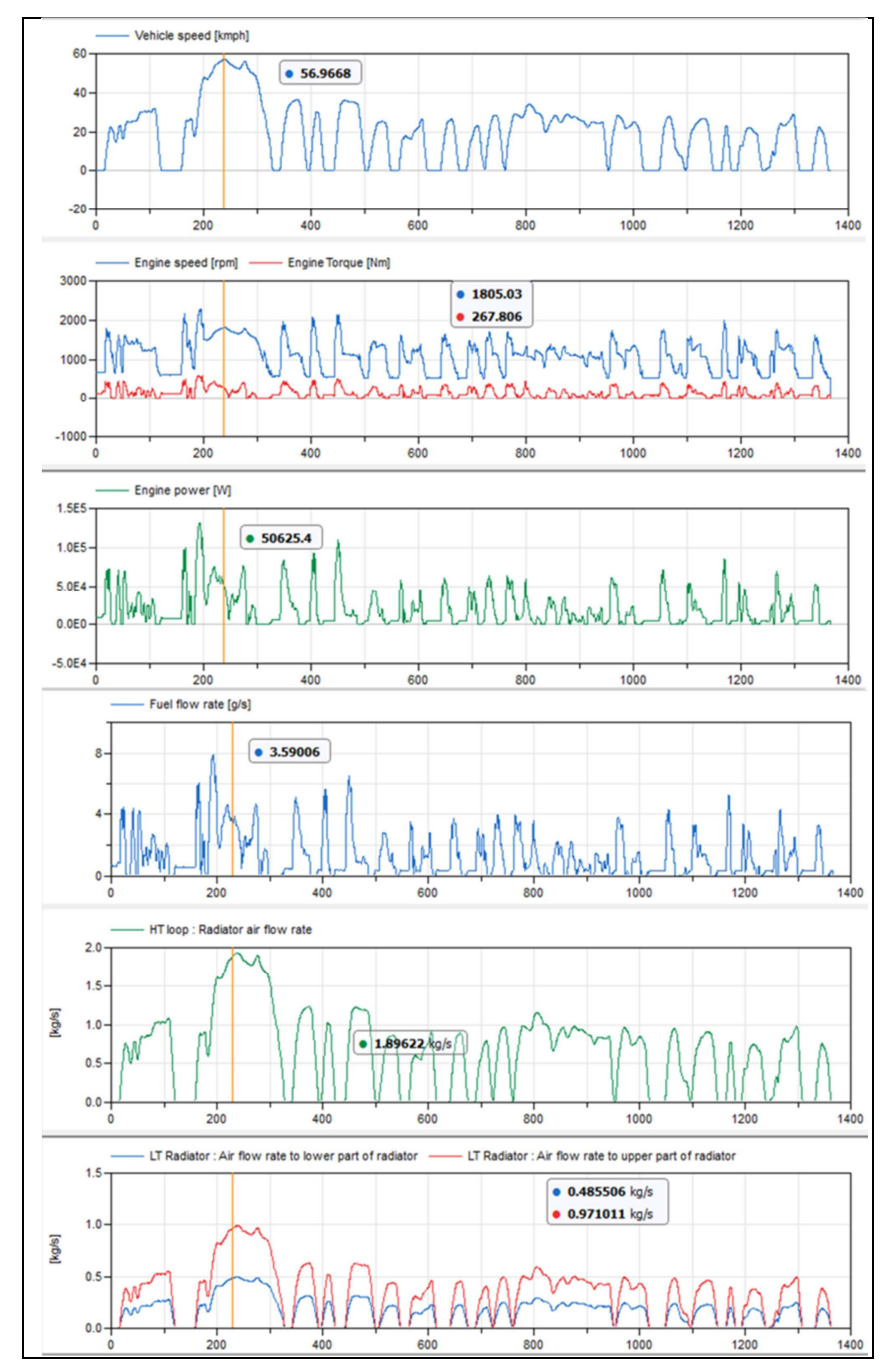

Figure 7. Operating conditions for FTP cycle

\subsection{Baseline Electric Pump Controller}

The electric pump controller is based on target coolant temperatures for various parts of the system. Unlike the mechanical pump system, the electric pump system can operate at lower pump speeds and flowrates. However, effectiveness of coolers can be low at low flowrates. With low effectiveness, there is minimal heat extracted in the cooler and thus no real effect on the coolant temperature. In this situation, the temperature of the fluid being cooled rises, but the electric pump controller will not respond to increase the flowrate since it is based on coolant temperature targets. Thus, a series of simulations were run to identify the appropriate minimum pump speed to ensure that the engine oil and transmission oil temperatures are kept under control. Figure 8 shows a comparison of the engine oil and transmission oil temperatures for the mechanical pump system and electric pump system at different minimum pump speeds. Based on these simulations, the minimum pump speed is set to 2000 RPM for the high temperature electric pump and 3000 RPM for the low temperature electric pump to provide similar temperatures as in the baseline mechanical pump system.

Figure 9 compares coolant temperatures in and out of the high temperature radiator along with the thermostat opening and mass flowrate through the radiator for the mechanical and electric pump systems. The coolant profiles are very similar.

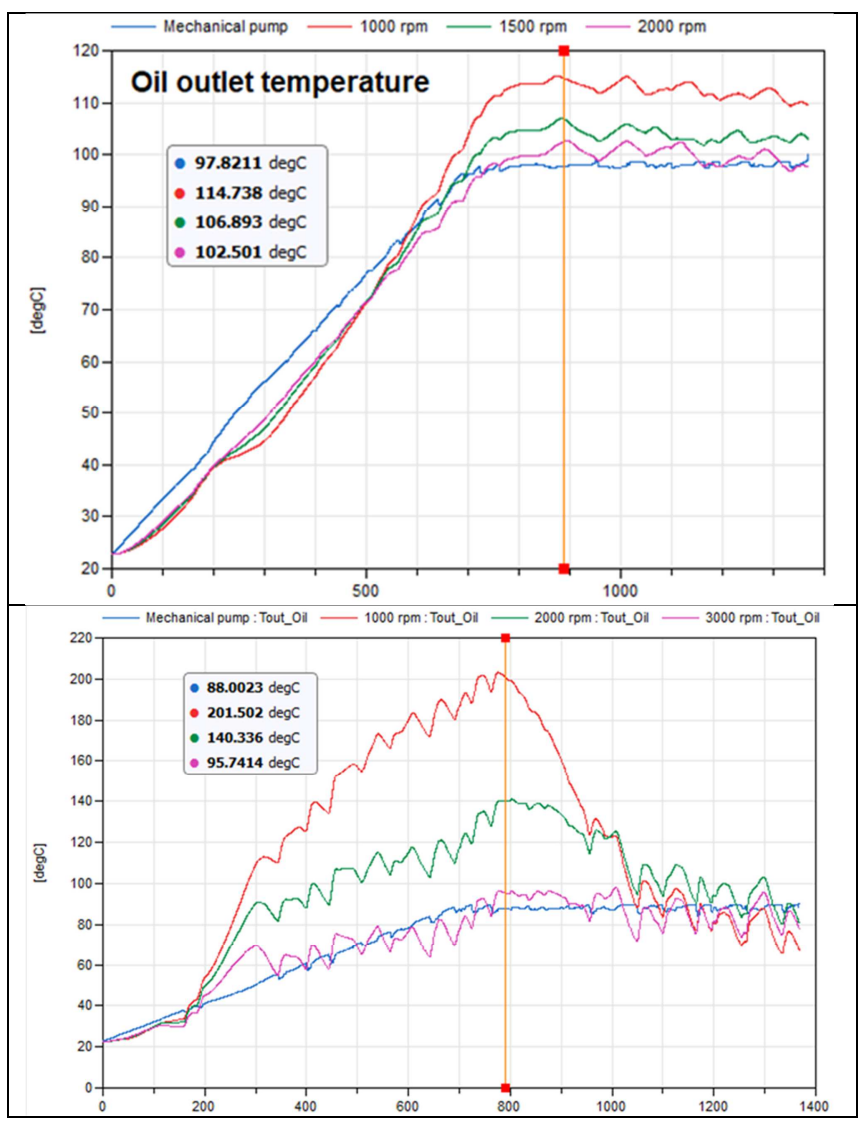

Figure 8. Effect of minimum electric pump speed on engine and transmission oil temperatures
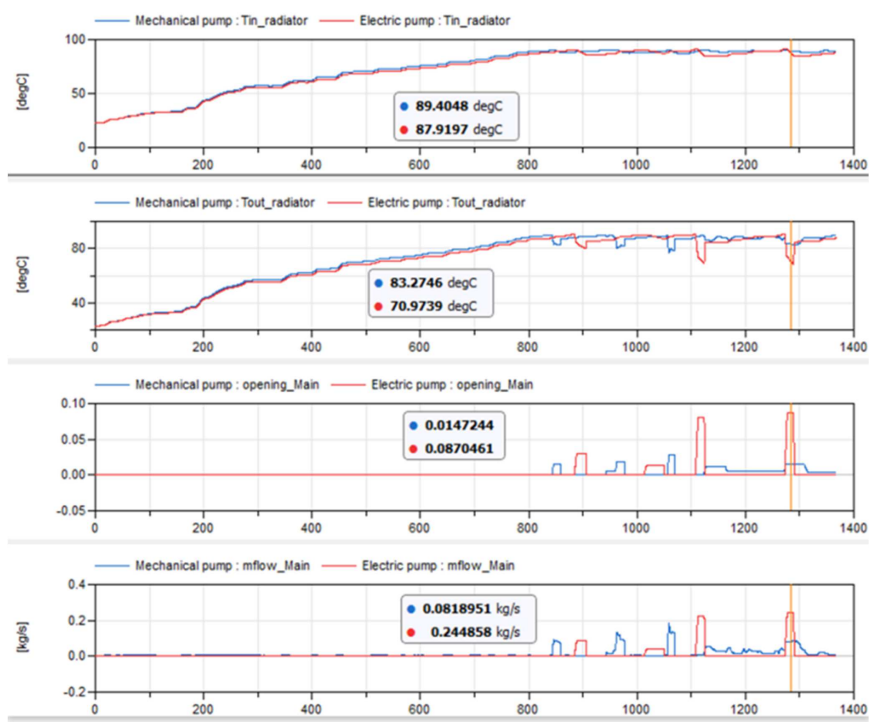

Figure 9. Comparison of coolant temperatures and flowrates at high temperature radiator 
Figure 10 compares high temperature pump conditions between the mechanical and electric pump systems. Since the FTP cycle is such a lightly loaded cycle, the electric pump system can operate at the minimum pump speed for the entire cycle. A comparison of the pump flowrates shows how much flow circulates in the mechanical pump system due to the linking of pump speed with engine speed. Even though the overall efficiency of the electric pump system is lower than that of the mechanical pump system, the total pump energy is significantly less in the electric pump system as the hydraulic power requirement is so much lower due to the lower flowrates.

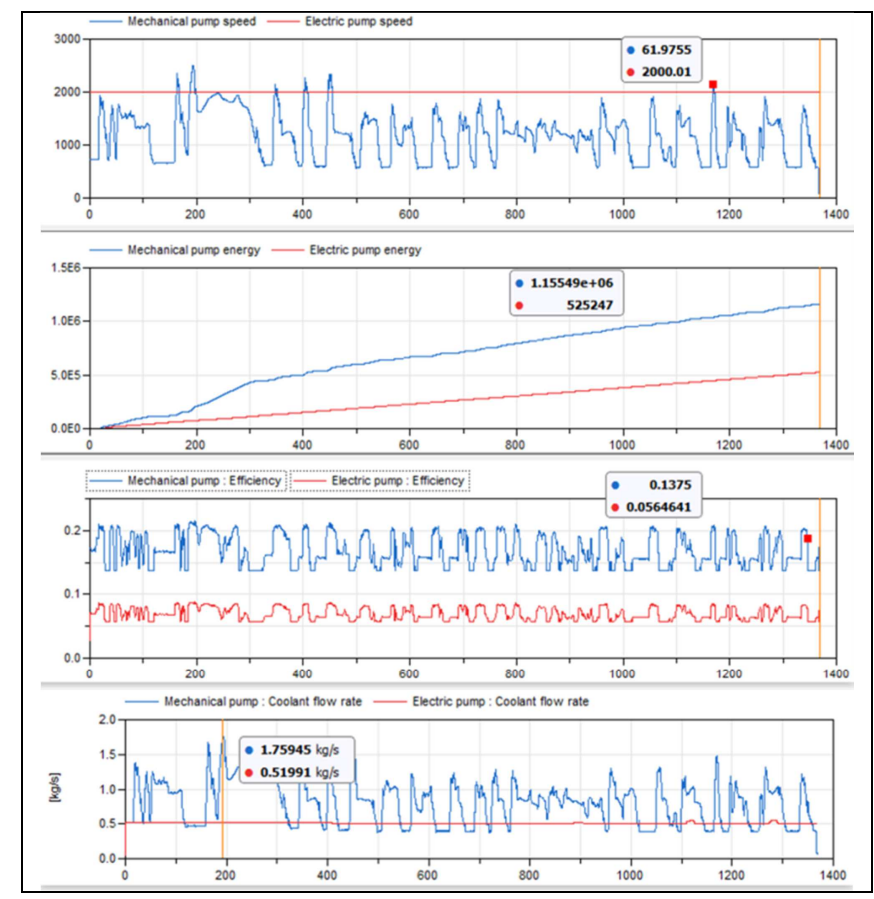

Figure 10. Comparison of high temperature pump conditions for mechanical and electric pump systems

Figure 11 compares temperatures in the low temperature circuit between the mechanical and electric pump systems. Temperatures are similar between the two systems though slightly higher in the electric pump system.

Figure 12 compares low temperature pump conditions between the mechanical and electric pump systems. Again, the electric pump system can run at the minimum pump speed for the entire cycle. As with the high temp pump, the overall flowrates are significantly less in the electric pump system. The overall efficiency of the electric pump system is again lower than the mechanical pump system. For the low temperature circuit, the total pump energy for the electric pump system is larger than the mechanical pump system as the reduction in hydraulic power is not enough to offset the reduced efficiency.
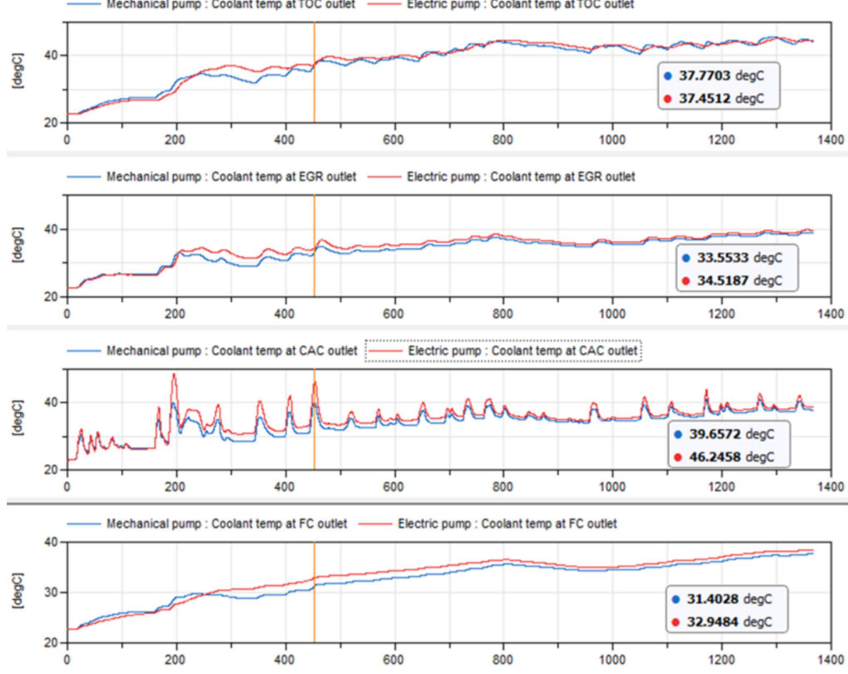

Figure 11. Comparison of temperatures in the low temperature circuit

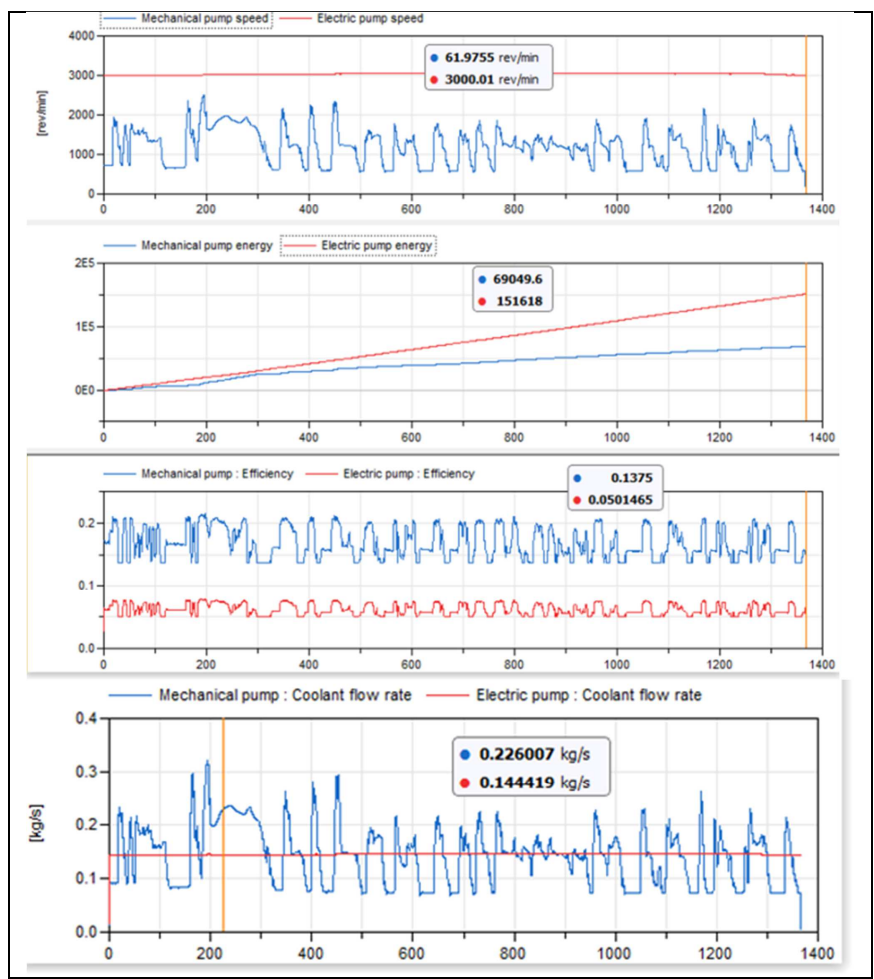

Figure 12. Comparison of low temperature pump conditions for mechanical and electric pump systems

Table 1 shows a comparison of the fuel consumption between the mechanical and electric pump systems. The fuel consumption on the cycle is reduced from $1.6 \%$ of the total cycle fuel to approximately $0.9 \%$ of the total cycle fuel with the electric pump system. As discussed previously, the electric pump system provides a benefit on the high temperature circuit but not on the low temperature circuit due to the flowrate required to manage the transmission oil temperatures given that the mechanical pump system is inherently more efficient. 
Table 1. Fuel consumption comparisons for the mechanical and electric pump systems

\begin{tabular}{|c|c|c|}
\hline Vehicle & $\begin{array}{c}\text { Fuel consumption } \\
{[\mathrm{g}]}\end{array}$ & $\begin{array}{c}\text { \% of total fuel } \\
\text { consumption }\end{array}$ \\
\hline $\begin{array}{c}\text { HT Mechanical } \\
\text { pump }\end{array}$ & 26.62 & 1.5 \\
\hline HT Electric pump & 12.10 & 0.69 \\
\hline $\begin{array}{c}\text { LT Mechanical } \\
\text { pump }\end{array}$ & 1.591 & 0.090 \\
\hline \begin{tabular}{c} 
LT Electric pump \\
\hline
\end{tabular} & 3.493 & 0.198 \\
\hline
\end{tabular}

\subsection{Electric Pump Controller with Oil-Based Control}

As described in Section 2.3, the baseline electric pump controller is based on target coolant temperatures. However, this control scheme requires that the minimum pump speed is set to control oil temperatures indirectly. As seen in the previous section, the electric pump system basically operates at minimum pump speed due to the light loads in the FTP cycle.

Another potential benefit of the electric pump system is the ability to accelerate the warmup of the engine and transmission oil by controlling the flow to the oil coolers. This benefit translates into fuel consumption due to reduced losses and friction due to oil temperatures that more rapidly reach the desired operating range. To evaluate this potential, the control algorithm was modified to explicitly control the engine and transmission oil temperatures in addition to target coolant temperatures for the other coolers. Figure 13 shows a comparison between the two different electric pump control strategies for the engine and transmission oil temperatures. By explicitly considering the oil temperatures in the electric pump strategy, the electric pump system can deliver oil temperatures that more quickly reach desired operating range while still managing the maximum temperature constraints.

Though the additional steps to translate these operating temperature benefits into fuel consumption metrics were beyond the scope of this work, they are being considered for further development of the simulation platform for Hanon. Potential future work also includes simulation of different vehicle cycles to evaluate fuel economy potential on a wider range of relevant usage profiles.

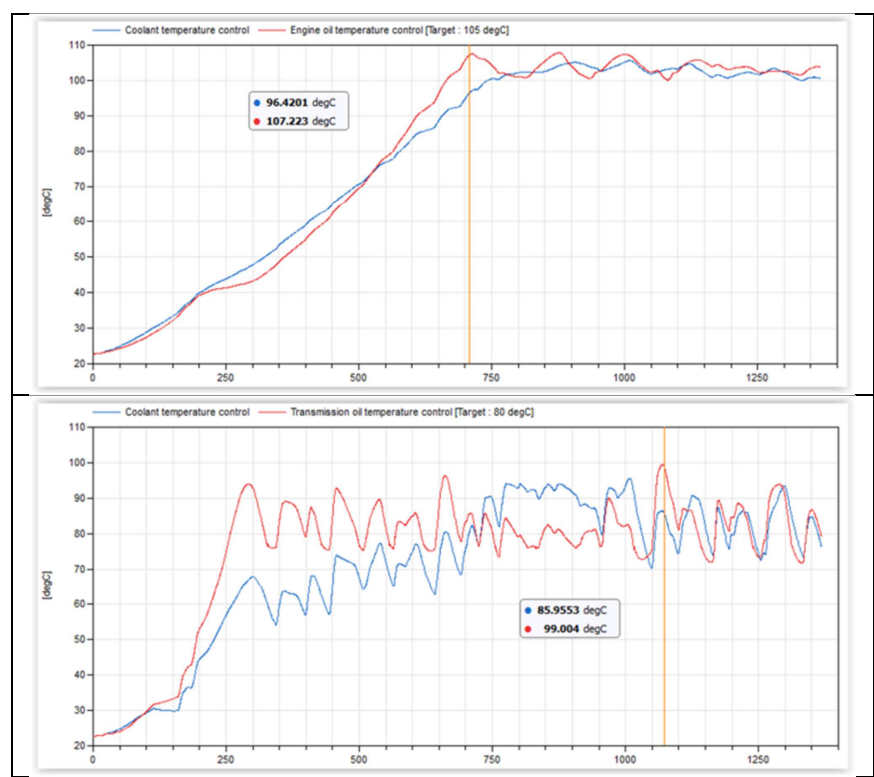

Figure 13. Comparison of electric pump systems with coolant only and coolant + oil temperature control, engine and transmission oil

\section{Summary}

This paper describes an effort to develop an analytic platform for Hanon Systems to evaluate the electrification potential for powertrain cooling systems. This analytic platform allows rapid virtual prototyping of different cooling systems to evaluate the potential of Hanon hardware and controls solutions for systems optimization. This platform was demonstrated on a model of the diesel cooling system for the Ford Power Stroke diesel 6.7L V8. A baseline model of the mechanical pump system was built and characterized for flow and thermal response. An electric pump variant of the system was built by replacing the mechanical pump with electric pumps from Hanon. Two different electric pump control strategies were implemented. The electric pump system demonstrated a fuel economy benefit when evaluated on the FTP cycle and also showed the potential benefit for more rapid warmup of engine and transmission oil with a modified control algorithm.

Future work on this model includes opportunities for better system characterization if data on the actual system can be obtained. In particular, actual characterization of the mechanical pump and heat exchanges would greatly improve model accuracy. Quantifying the benefits of the increased warmup of the oil temperatures for reduction in friction and losses is also a focus of future work. Integrating the cooling system model with a full vehicle simulation would reduce the need for driving the simulations with engine conditions and pick up additional interactions with the vehicle loads. Simulation on different vehicle cycles would also allow the evaluation of fuel economy potential on a wider range of usage profiles. 
While this work focused on simply replacing the mechanical pump with electric pumps, future work with this modeling capability includes evaluation of concepts to redesign the system and develop control strategies to take full advantage of the capability of the electric pump system to deliver flow on demand to individual coolers in the high and low temperature circuits.

\section{Acknowledgements}

The authors would like to thank Chris Leonello and Ondrej Pavlica from Hanon Systems for providing surrogate heat exchanger data to support thermal characterization of the system.

\section{References}

Arvind Thiruvengadam, Saroj Pradhan, Pragalath Thiruvengadam, Marc Besch, Daniel Carder, and Oscar Delgado. Heavy-Duty Vehicle Diesel Engine Efficiency Evaluation and Energy Audit, Final Report, October 2014, https://www.theicct.org/sites/default/files/publications/HD V_engine-efficiency-eval_WVU-rpt_oct2014.pdf

John Batteh, Jesse Gohl, Sureshkumar Chandrasekar. Integrated Vehicle Thermal Management in Modelica: Overview and Appliations. Proceedings of the $10^{\text {th }}$ International Modelica Conference, March 10-12, 2014 , Lund, Sweden. doi: 10.3384/ECP14096409.

C. Bouvy, P. Jeck, J. Gissing, T. Lichius, L. Ecksterin. Holistic Vehicle Simulation using Modelica - An Application on Thermal Management and Operation Strategy for Electrified Vehicles. Proceedings of 9th International Modelica Conference, pp. 263-270, 2012.

Deraad, S., Fulton, B., Gryglak, A., Hallgren, B. et al., "The New Ford 6.7L V-8 Turbocharged Diesel Engine," SAE Technical Paper 2010-01-1101, 2010, https://doi.org/10.4271/2010-01-1101.

I. Krüger, A. Mehlhase and G. Schmitz. Energy Consumption of Battery Cooling In Hybrid Electric Vehicles. Proceedings of 14th International Refrigeration and Air Conditioning Conference, 2012.

Modelon AB, Lund, Sweden. (2018). Heat Exchanger Library. http://www.modelon.com/products/modelonlibrary-suite/heat-exchanger-library/

Modelon AB, Lund, Sweden. (2018). Liquid Cooling Library. $\quad$ http://www.modelon.com/products/modelonlibrary-suite/liquid-cooling-library/

Massimo Stellato, Luca Bergianti, and John Batteh. Powertrain and Thermal System Simulation Models of a High Performance Electric Road Vehicle. Proceedings of the $12^{\text {th }}$ International Modelica Conference, May 15-17, 2017, Prague, Czech Republic. doi: 10.3384/ecp17132171.

Jason Zheng. Multi-Disciplinary Approach to Thermal Systems Design and Optimization. SAE Thermal Management Systems Symposium, 18TMSS-0012, October 9-11, 2018. San Diego, CA. 